\title{
Prospective analysis of obesity and the effect of bariatric surgery on urinary incontinence
}

\section{Análisis prospectivo de la obesidad y el efecto de la cirugía bariátrica en la incontinencia urinaria}

\begin{abstract}
(DIván Calvo-Vázquez, ${ }^{1 *}$ (DBDaudelio Rodríguez-Rodríguez, ${ }^{1}$ Erick Alejandro Hernández-Méndez, ${ }^{1}$ (D)Guadalupe Michel López-Bravo,, Mario Enrique Ortega-González, ${ }^{1}$ Pascual Cortes-Raygoza, ${ }^{1}$ Ulises Cristóbal Sánchez-Aquino, ${ }^{1}$ Gustavo Adolfo Véliz-Cabrera, ${ }^{1}$ (DCarlos Martínez-Arroyo, ${ }^{1}$ DGerardo Fernández-Noyola, ${ }^{1}$ Jorge Gustavo Morales-Montor, ${ }^{1}$ D Mauricio Cantellano-Orozco, ${ }^{1}$ iDCarlos Pacheco-Gahbler. ${ }^{1}$
\end{abstract}

\section{Abstract}

Objective: To determine the presence of urinary incontinence in obese women and the postoperative effect of bariatric surgery at months 3, 6, and 12 .

Materials and methods: An observational, prospective, and longitudinal study was conducted on obese women that underwent bariatric surgery within the time frame of April to July 2017. Inferential statistics with a bivariate analysis were performed using the chi-square test.

Results: Sixty-seven patients were included in the study. A total of $73.1 \%$ presented with some type of urinary incontinence. Their mean age was 42.7 years, mean preoperative weight was $108.3 \mathrm{~kg}$, and mean body mass index was 42.7 $\mathrm{kg} / \mathrm{m} 2$. The most frequent type of urinary incontinence was stress incontinence $(55.1 \%), 65.3 \%$ of the patients presented with mild urinary incontinence, and quality of life was negatively affected in $30.6 \%$. One year after surgery, mean body mass index was $30.9 \mathrm{~kg} / \mathrm{m} 2$ and $13.4 \%$ of the patients continued to present with urinary incontinence, all of which were cases of stress incontinence. The postoperative improvement of urinary incontinence was statistically significant in relation to the three types of incontinence $(\mathrm{p}=0.001$ ), frequency $(\mathrm{p}<0.001)$, and quality of life $(\mathrm{p}<0.001)$. Urinary incontinence was completely

Keywords:

Urinary incontinence, Obesity, Stress incontinence, Urge incontinence, Mixed incontinence.

Correpondencia:

* Iván Calvo-Vázquez, Scala Residencial Calzada México-Xochimilco 4850, Departamento D-503, Arenal de Guadalupe,

CP 14389. Correo electrónico: dr.ivancv unam@hotmail.com resolved in $81.6 \%$ of the patients.

Conclusions: Weight loss after bariatric surgery greatly resolved urinary incontinence, showing that weight reduction should be considered a first-line therapy for managing that syndrome. The primary aim of bariatric surgery is to reduce cardiovascular morbidity, but the recovery from and/or improvement of urinary incontinence should be routinely evaluated, offering another important long-term benefit.

Citación: Calvo-Vázquez I.C., Rodríguez-Rodríguez B., Hernández-Méndez E.A., López-Bravo G. M., Ortega-González M.E., Cortés-Raygoza P., Sánchez-Aquino, U.C., Véliz-Cabrera G.A., Martínez-Arroyo C., Fernández-Noyola G., Morales-Montor J.G., Cantellano-Orozco M., Pachecho-Gahbler C. Análisis prospectivo de la obesidad y el efecto de la cirugía bariátrica en la incontinencia urinaria. Rev. Mex. Urol. 2019;79(3):pp. 1-12.

${ }^{1}$ Hospital General “Dr. Manuel Gea González”, División de Urología, Ciudad de México, México.

Recepción: 28 de octubre de 2018.

Aceptación: 16 de marzo de 2019. 


\section{Análisis prospectivo de la obesidad y el efecto de la cirugía.... Calvo-Vázquez l., et al.}

Palabras clave: Incontinencia urinaria, obesidad, incontinencia de esfuerzo, incontinencia de urgencia, incontinencia mixta.

\section{Resumen}

Objetivo: Determinar la incontinencia urinaria (IU) en mujeres con obesidad y el efecto 3, 6 y 12 meses después de la cirugía bariátrica. Materiales y Métodos: Entre abril y julio de 2017 se realizó un estudio observacional, prospectivo y longitudinal en mujeres con obesidad, sometidas a cirugía bariátrica, realizando análisis estadístico inferencial y bivariado mediante Chi Cuadrada.

Resultados: Sesenta y siete pacientes en total, de las cuales 73.1\% tenían algún tipo de IU. Edad de 42.7 años, peso prequirúrgico $108.3 \mathrm{~kg}$ e IMC de $42.7 \mathrm{~kg} / \mathrm{m} 2$. La incontinencia más frecuente fue de esfuerzo $(55.1 \%)$, la IU leve apareció en el $65.3 \%$ y la afección en la calidad de vida en el $30.6 \%$. Un año después, el IMC fue de $30.9 \mathrm{Kg} / \mathrm{m} 2$, con una persistencia de iu del $13.4 \%$, todas de esfuerzo. La mejoría postquirúrgica de iv fue estadísticamente significativa para los tres tipos $(\mathrm{p}=0.001)$, frecuencia $(\mathrm{p}<0.001)$ y en la calidad de vida $(\mathrm{p}<0.001)$, obteniendo una resolución completa de $81.6 \%$.

Conclusiones: Confirmamos que la pérdida de peso posterior a la cirugía bariátrica tiene una gran resolución de la IU, debiendo considerarse como una terapia de primera línea. Aunque los objetivos principales de esta cirugía son disminuir las morbilidades cardiovasculares, la recuperación y/o mejoría de la IU debería ser evaluada rutinariamente, ofreciendo otro beneficio a largo plazo.

\section{Antecedentes}

La obesidad es un importante problema de Salud Pública, sobre todo en países industrializados, predispone a muchas enfermedades y reduce la expectativa de vida. ${ }^{(1)}$ De hecho, la obesidad se ha incrementado durante las últimas tres décadas y aumentará aún más en los próximos 20 años. Esta carga sanitaria y económica, considerada exclusiva del mundo industrializado, se está extendiendo a los países en desarrollo a medida que su dieta y estilo de vida se han occidentalizado. ${ }^{(2)}$

La obesidad afecta a más de un tercio de la población adulta estadounidense, ${ }^{(3)}$ y está asociada a un mayor riesgo de comorbilidades metabólicas, cardiovasculares, carcinógenas y deterioro de la calidad de vida. ${ }^{(4)}$

Más de la mitad de las mujeres estadounidenses tienen sobrepeso (IMC de 25 a $29.9 \mathrm{~kg} / \mathrm{m}^{2}$ ) o son obesas (IMC de $30 \mathrm{Kg} / \mathrm{m} 2$ o más); de hecho, la prevalencia de obesidad está aumentando casi 6\% anual. ${ }^{(5)}$ En México, según la Encuesta Nacional de Salud y Nutrición (ENSANUT, 2016) la prevalencia combinada de sobrepeso y obesidad en mujeres adultas es de $75.6 \%$, este incremento es mayor en zonas rurales (aumento de $8.4 \%$ ) que en zonas urbanas (aumento de 1.6\%). ${ }^{(6)}$ 
La obesidad es un importante factor etiológico modificable que se asocia con varias enfermedades, incluyendo enfermedad cardiovascular -la causa de muerte más común-; trastornos músculo esqueléticos, Diabetes Mellitus y algunos tipos de cáncer (colon, endo-

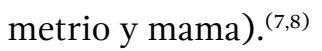

Basado en la definición del Índice de Masa Corporal (IMC), los valores se consideran de la siguiente manera: peso insuficiente en $<18.49$ $\mathrm{kg} / \mathrm{m} 2$, peso normal de $18.5-24.99 \mathrm{~kg} / \mathrm{m} 2$, sobrepeso de $25-29.99 \mathrm{~kg} / \mathrm{m} 2$ y >30 kg/m2 como obesidad, la Organización Mundial de la Salud, estima que 1.9 millones y 600 millones de adultos de 18 años tienen sobrepeso y obesidad, respectivamente.

La obesidad es un factor de riesgo establecido y modificable para la Incontinencia Urinaria (IU), pero los datos analíticos y comprehensivos sobre el efecto de la pérdida de peso en diversos aspectos de los trastornos del suelo pélvico, incontinencia urinaria, disfunción sexual femenina o enfermedad específica cardiovascular son deficientes. ${ }^{(9)}$

Se aumentan los costos sanitarios y de prevención. Pocos estudios han establecido que la obesidad promueve todos los tipos de Incontinencia Urinaria (IU) y trastornos del piso pélvico. ${ }^{(10)}$ Basándose en una revisión sistemática de los datos reportados entre 1990 y 2009 , Withrow y Alter estimaron que los tratamientos de estos y otros problemas relacionados con la obesidad representan entre 0.7 y $2.8 \%$ del total de los gastos mundiales de salud. ${ }^{(11)}$

La obesidad también se ha asociado con el desarrollo de Incontinencia Urinaria (IU), que no sólo reduce la calidad de vida, sino también aumenta los gastos médicos; investigaciones anteriores han asociado un aumento de 5 puntos en el IMC con un aumento del $20-70 \%$ de riesgo de IU. ${ }^{(12)}$ La Diabetes Mellitus, como otra condición asociada a la obesidad, también puede aumentar el riesgo de IU como resultado de la hiperactividad del detrusor. ${ }^{(5,13)}$

La prevalencia de la IU se incrementa con la edad, con tasa típica en la población joven (20-30\%), aumento de la etapa adulta (prevalencia de 30 a $40 \%$ ) e incremento sostenido en la población de mayor edad (prevalencia de 30 a $50 \%) .{ }^{(14)}$ La incontinencia urinaria de esfuerzo es el tipo más frecuente de las mujeres entre 25 y 65 años de edad. Gran parte de las mujeres que padecen incontinencia urinaria no buscan atención médica, 25\% en Estados Unidos y 33\% en Europa. ${ }^{(15,16)}$

Las mujeres con IU presentan mayor índice de masa corporal que las mujeres con continencia urinaria normal. La incontinencia urinaria tiene mas prevalencia en mujeres (49.3\%) que en hombres (21.8\%). ${ }^{(12)}$

Los tipos de IU, definida como pérdida involuntaria de orina, son la incontinencia urinaria de esfuerzo (IUE), la incontinencia urinaria de urgencia (IUU) y la incontinencia urinaria $\operatorname{mixta}(\mathrm{IUM}){ }^{(17)}$

La IUE, también conocida como incontinencia relacionada con la actividad, es la pérdida involuntaria de orina en el esfuerzo físico, en ausencia de contracciones de la vejiga. La IUU es la pérdida de orina cuando una necesidad fuerte y repentina de orinar, causa espasmo vesical. La IUM es la pérdida involuntaria de orina asociada con urgencia, así como con el esfuerzo físico. El tratamiento depende del tipo de incontinencia, pero todos los tipos parecen beneficiarse de la restricción de líquidos, la regulación de diuréticos y otros medicamentos, ejercicios de Kegel, así como de la pérdida de peso. ${ }^{(18)}$

El tratamiento de la incontinencia urinaria en pacientes obesos comienza con reducción 
de peso, con una dieta adecuada en calorías y la corrección de los trastornos alimentarios. La tasa de fracaso de las mujeres con un IMC >35 $\mathrm{kg} / \mathrm{m} 2$ es muy alta, $81 \%$ alcanzan la continencia, en comparación con el $92 \%$ al $96 \%$ en la población general. ${ }^{(19,20)}$

El papel de la cirugía bariátrica en la incontinencia urinaria y el mejoramiento de la calidad de vida sigue siendo poco estudiado. Los trastornos del piso pélvico, la incontinencia urinaria, el prolapso de órganos pélvicos y la incontinencia fecal, afectan de 50.9 al $75 \%$ de las mujeres que se someten a cirugía de pérdida de peso y en otras series del $60-70 \%$, con una prevalencia del $28 \%$ para el tipo de IUE, $4 \%$ IUU y $32 \%$ para IUM y en otras series $37.7 \%$ IUU y $59 \%$ IUE. ${ }^{(21-23)}$

Se sabe que los pacientes obesos tienen un aumento de la presión intraabdominal, lo que contribuye a varias comorbilidades asociadas. Esta asociación reconocida ha llevado a la investigación y la demostración del efecto positivo de la pérdida de peso, especialmente la incontinencia urinaria. ${ }^{(24)}$

Etiológicamente son cuatro los factores que se asocian con la obesidad y que, a su vez, aumentan el riesgo de IU: aumento de la grasa abdominal, lo cual aumenta la presión intravesical, hipermovilidad uretral y aumento de la presión abdominal, que causan inestabilidad del detrusor y hernia de disco intervertebral, que afecta la inervación de la vejiga. ${ }^{(25)}$ Estudios urodinámicos apoyan la existencia de estos procesos, encontrando que la pérdida de peso conduce a la disminución de la presión intravesical y al aumento de la capacidad cistométrica. ${ }^{(26,27)}$

\section{Materiales y métodos}

Entre abril y julio de 2017, en el Hospital General "Dr. Manuel Gea González", se llevó a cabo un estudio prospectivo, analítico y longitudinal en el cual se analizaron a todas las mujeres con obesidad mayores de 20 años sometidas a cirugía bariátrica.

\section{Criterios de exclusión}

Historia de procedimientos anti-incontinencia antes y/o después del inicio del estudio, el uso de fármacos para incontinencia urinaria (por ejemplo, anticolinérgico), historia de enfermedades neurológicas, embarazo y pérdida de seguimiento en los cuestionarios del tiempo establecido.

La prevalencia de la realización de cirugía bariátrica en mujeres con obesidad en el Hospital General "Dr. Manuel Gea González” es de: $5 \%$. Por lo tanto, la determinación de la muestra se realizó con la fórmula de estimación de proporciones. $\mathrm{N}=\mathrm{z} 2 \mathrm{pq} / \mathrm{d} 2$. El número de pacientes que se incluyeron en la muestra fue de 72. El tamaño de la población de mujeres con obesidad que fueron tratadas con cirugía bariátrica en un año fue de 60 . Se acepta un margen de error de $5 \%$ con un nivel de confianza de $95 \%$, por lo que el tamaño de la muestra que se recomienda para este estudio es de $53 \%$.

Tomando en cuenta las siguientes variables: Edad, peso, talla, IMC, tabaquismo, número de embarazos y partos, antecedente de cirugía bariátrica previa, cirugía pélvica, tipo de cirugía bariátrica, comorbilidades (DM y HAS), registro de peso e IMC reducido post a tratamiento bariátrico. 
Incontinencia urinaria prevalente se definió como por lo menos un episodio de incontinencia urinaria a la semana y remisión se definió como cambio de la incontinencia urinaria prevalente en la línea de base a menos de un episodio semanal. Se clasificó como incontinencia de esfuerzo, urgencia o mixta.

La disminución en el número de episodios de incontinencia se reportó como "disminución de la incontinencia”, cuando no informaron incontinencia se reportó como "remisión completa”. Por su parte, el cambio de más a menos frecuente se consideró como "mejoría".

El índice de masa corporal se calculó como el peso en kilogramos dividido por altura en metros cuadrados. El cambio de peso se calculó como el cambio porcentual con respecto al valor basal.

Al menos un cuadro de incontinencia semanal se asocia con la reducción de la calidad de vida.

El consumo de tabaco se definió como negativo si el término del consumo fue hace más de 10 años.

La presencia de DM se definió como nivel de glicemia en sangre $>126 \mathrm{mg} / \mathrm{dl}$, hemoglobina glucosilada $>7 \%$ y/o uso de medicamentos para DM. La presencia de HAs se definió como presencia de presión arterial sistólica $>140 \mathrm{mmHg}$, presión diastólica $>90 \mathrm{mmHg}$ y/o uso de cualquier medicamento antihipertensivo.

\section{Descripción de procedimiento}

Los pacientes completaron la forma corta del cuestionario de consulta internacional sobre incontinencia urinaria (ICIQ-UI-SF) y el cuestionario de impacto de incontinencia (IIQ-7).
Ambos cuestionarios miden la gravedad de la IU y su impacto en la calidad de vida. Lo anterior se realizó previo a la intervención quirúrgica y a los 3, 6, 9 y 12 meses posteriores a la cirugía. El diagnóstico de IUE o IUU se basó en la respuesta a la pregunta 6 del ICIQ-UI-SF.

\section{Resultados}

Se obtuvo un total de 72 pacientes de los cuales 67 contestaron todos los cuestionarios, la edad media encontrada fue de $40.46+/-9.1$ años, con un peso prequirúrgico promedio de 107.6 +/-20.6 kg e IMC de $42.02+/-7.3 \mathrm{~kg} / \mathrm{m} 2$.

El 73.1\% (49) de las pacientes tenían algún tipo o grado de incontinencia urinaria. De este grupo de mujeres incontinentes la media de edad fue $42.7+/-7.9$ años, peso prequirúrgico de $108.3+/-23.2 \mathrm{~kg}$, IMC de $42.7+/-8.3 \mathrm{~kg} / \mathrm{m} 2$ y talla media de $1.59+/-0.06$ metros (Tabla 1 ).

Tabla 1. Medias, valores mínimos y máximos de las variables cuantitativas prequirúrgicas

\begin{tabular}{lrr} 
& Media & \multicolumn{1}{c}{ Min.-Max. } \\
Edad & 42.7 & $25-56$ \\
Peso (Kg) & 108.3 & $76-187$ \\
Talla (m) & 1.59 & $1.45-1.72$ \\
IMC (Kg/m2) & 42.7 & $33.1-66.2$ \\
\hline
\end{tabular}

El porcentaje de mujeres con diagnóstico de DM y Has fue de $12.2 \%$ (6) para cada comorbilidad, la resistencia a la insulina se encontró en el $22.4 \%$ (11), hipotiroidismo $10.2 \%$ (5) y dislipidemia $8.2 \%$ (4). Solo $4.1 \%$ (2) contaba con antecedente de cirugía bariátrica previa, ninguna tenía cirugía pélvica para incontinencia, y $28.6 \%$ (14) de las pacientes fumaba (Tabla 2). 


\section{Análisis prospectivo de la obesidad y el efecto de la cirugía.... Calvo-Vázquez l., et al.}

Tabla 2. Características demográficas $(\mathrm{N}=49)$

\begin{tabular}{lcc} 
& $n$ & $\%$ \\
Comorbilidades & 6 & 12.2 \\
DM & 6 & 12.2 \\
HAs & 11 & 22.4 \\
Resistencia a la & & \\
insulina & 5 & 10.2 \\
Hipotiroidismo & 4 & 8.2 \\
Dislipidemia & 2 & 4.1 \\
Cirugía previa & 14 & 28.6 \\
Tabaquismo & & \\
Tipo de cirugía & 26 & 53.1 \\
Manga gástrica & & \\
laparoscópica & 23 & 46.9 \\
Bypass gástrico & 49 & 73.1 \\
Incontinencia & & \\
Urinaria & 27 & 55.1 \\
Esfuerzo & 16 & 32.7 \\
Urgencia & 6 & 12.2 \\
Mixta & & \\
\hline
\end{tabular}

La incontinencia de urgencia representó $32.7 \%$ (16), de esfuerzo $55.1 \%$ (27) y mixta el $12.2 \%$ (6). El tipo de cirugía que más se realizó fue manga gástrica laparoscópica en 26 (53.1\%) pacientes, seguida de bypass gástrico laparoscópico en 23 (46.9\%) (Tabla 2).

La frecuencia con la que se presentaba la incontinencia prequirúrgica fue de $4.1 \%$ para continua, varias veces al día $4.1 \%$, diario $16.3 \%$, $2-3$ veces a la semana $8.2 \%$ y una vez por semana de $67.3 \%$. La cantidad moderada de orina pérdida se encontró en el $34.7 \%$ (17) y poca en $65.3 \%$ (32). La afección de la incontinencia en la calidad de vida solo se presentó en $30.6 \%$ (15) contra $69.4 \%$ (34) donde no repercutió.
La media de peso a los tres meses fue de $89.7 \mathrm{~kg} ; 81.6 \mathrm{~kg}$ a los 6 meses y de $78.3 \mathrm{~kg}$ a los 12 meses, con un IMC promedio de $35.4 \mathrm{~kg} /$ $\mathrm{m} 2$ a los 3 meses, $31.9 \mathrm{~kg} / \mathrm{m} 2$ a los 6 meses y de $30.9 \mathrm{~kg} / \mathrm{m} 2$ a los 12 meses. La pérdida de peso promedio a los tres meses fue de $18.55+$ /$7.8 \mathrm{Kg}, 26.6+/-11.1 \mathrm{~kg}$ a $\operatorname{los} 6$ y $30+/-11.3 \mathrm{Kg}$ al año, teniendo una disminución del IMC a los tres meses de $7.2+/-2.8 \mathrm{~kg} / \mathrm{m} 2,10.7+/-4.4 \mathrm{~kg} /$ $\mathrm{m} 2$ a los 6 meses y $11.8+/-4.1 \mathrm{~kg} / \mathrm{m} 2$ al año postquirúrgico (Tabla 3 ).

Tabla 3. Medias, valores mínimos y máximos del peso perdido e IMC recuperado a los 3,6 y 12 meses

$\begin{array}{lcc} & \text { Media } & \text { Min.-Max. } \\ \text { Peso (Kg) } & & \\ 3 \text { meses } & 18.55+/-7.8 & 6.9-41.5 \\ 6 \text { meses } & 26.6+/-11.1 & 10.9-63 \\ 12 \text { meses } & 30+/-11.3 & 10.9-62 \\ \text { IMC (Kg/m2) } & & \\ 3 \text { meses } & 7.2+/-2.8 & 2.6-14.71 \\ 6 \text { meses } & 10.7+/-4.4 & 4.25-22.33 \\ 12 \text { meses } & 11.8+/-4.1 & 4.26-22.06\end{array}$

A los tres meses del procedimiento quirúrgico las pacientes que continuaban con incontinencia urinaria fueron $28(41.8 \%)$, representadas en IUU $32.1 \%$ (9), IUE $53.6 \%$ (15) е IUM $14.3 \%$ (4). La frecuencia se encontró con pérdidas diarias en 4 (14.3\%) de las pacientes, 2-3 veces por semana en 11 (39.3\%), una vez por semana en $13(46.4 \%)$ y ninguna para pérdidas continuas ni varias veces al día. La cantidad moderada se presentó en $6(21.4 \%)$ y poca en $22(78.6 \%)$, teniendo persistencia en la afección de la calidad de vida en solo 10 (35.7\%) (Tabla 4). 
A los seis meses la persistencia de la incontinencia urinaria solo fue del $17.9 \%$ (12), encontrando IUE $83.3 \%$ (10) e IUM $16.7 \%$ (2), la frecuencia se encontró diario para una paciente (8.3\%), 2-3 veces a la semana en $16.7 \%$ (2) y una vez a la semana $75 \%$ (9), ya no se encontraron presentes las demás frecuencias. La cantidad moderada se presentó en 2 (16.7\%) pacientes y poca en 10 (83.3\%), solo 6 (50\%) continuaba con afectación en la calidad de vida (Tabla 4 ).

Tabla 4. Variables pre y postquirúrgicas, 3 y 6 meses después de la cirugía

\begin{tabular}{lccccc}
\multicolumn{1}{c}{ Variable } & Prequirúrgica & 3 meses & 6 meses & 12 meses & $p$ \\
Incontinencia & $73.1 \%(49)$ & $41.8 \%(28)$ & $17.9 \%(12)$ & $13.4 \%(9)$ & 0.02 \\
Tipo & & & & & 0.001 \\
IUU & $32.7 \%(16)$ & $32.1 \%(9)$ & 0 & 0 & \\
IUE & $55.1 \%(27)$ & $53.6 \%(15)$ & $83.3 \%(10)$ & $100 \%(9)$ & \\
IUM & $12.2 \%(6)$ & $14.3 \%(4)$ & $16.7 \%(2)$ & 0 & \\
Frecuencia & & & & & $<0.001$ \\
Continua & $4.1 \%(2)$ & 0 & 0 & 0 & \\
Varias veces al día & $4.1 \%(2)$ & 0 & 0 & 0 & \\
Diaria & $16.3 \%(8)$ & $14.3 \%(4)$ & $8.3 \%(1)$ & $11.1 \%(1)$ & \\
$2-3$ veces por semana & $8.2 \%(4)$ & $39.3 \%(11)$ & $16.7 \%(2)$ & 0 & \\
1 vez por semana & $67.3 \%(33)$ & $46.4 \%(13)$ & $75 \%(9)$ & $88.9 \%(8)$ & \\
Cantidad & & & & & \\
Poca & $65.3 \%(32)$ & $78.6 \%(22)$ & $83.3 \%(10)$ & $88.9 \%(8)$ & \\
Moderada & $34.7 \%(17)$ & $21.4 \%(6)$ & $16.7 \%(2)$ & $11.1 \%(1)$ & \\
Afectación en calidad de vida & $30.6 \%(15)$ & $35.7 \%(10)$ & $50 \%(6)$ & $77.8 \%(7)$ & $<0.001$ \\
\hline
\end{tabular}

$\mathrm{Al}$ año de seguimiento, las pacientes que se mantenían con incontinencia urinaria fueron 9 (13.4\%), de estas el 100\% ocupó la IUE. La frecuencia diaria se continuó presentado en una paciente $(11.1 \%)$, seguido de una vez por semana en el resto $(88.9 \%)$, la cantidad de orina pérdida fue poca en la mayoría de ellas ( $88.9 \%)$, solo una paciente refirió persistir con cantidad moderada (11.1\%), la afección de la calidad de vida aún permanecía en $77.8 \%$ (7) (Tabla 4).

Mediante estadística inferencial y pruebas de chi cuadrada se calculó que existe mayor riesgo significativo en pacientes con parto vía vaginal, respecto de las pacientes que no lo tuvieron, toda vez que presentaron algún tipo de incontinencia $(\mathrm{p}=0.03), \mathrm{OR}=5.06, \mathrm{IC}=1.04-24.55$.
La tasa de incontinencia urinaria fue de $73.1 \%$ antes de la cirugía y de $13.4 \%$ a los 12 meses de seguimiento con $\mathrm{p}=0.02$, para los tres tipos de incontinencia con $\mathrm{p}=0.001$, frecuencia $\mathrm{p}<0.001$, cantidad $\mathrm{p}=0.117$ y para la afección en la calidad de vida con $\mathrm{p}<0.001$.

\section{Discusión}

La obesidad se asocia a varias enfermedades crónico degenerativas, incluyendo cardiovasculares, DM y dislipidemia. ${ }^{(7,8)}$ En este estudio encontramos que la comorbilidad mayormente asociada fue resistencia a la insulina en $22.4 \%$, seguida de DM y HAS en $12.2 \%$ cada una. Tam- 


\section{Análisis prospectivo de la obesidad y el efecto de la cirugía.... Calvo-Vázquez l., et al.}

bién se ha identificado como un factor de riesgo para incontinencia urinaria, al ser un problema de Salud Pública que va en ascenso, los síntomas urinarios relacionados también deberían de aumentar, disminuyendo la calidad de vida y aumentando los gastos a la salud.

Del total de nuestra población de estudio, solo 73.1\% presentaba algún tipo o grado de IU, mayor a la prevalencia encontrada por Subak et al. 49.3\%, ${ }^{(12)}$ Shimonov et al. de $37.7 \%,{ }^{(21)}$ y Said et al. de $50.9 \%{ }^{(22)}$ Sin embargo hay series que reportan una prevalencia de hasta $75 \%$ parecida a nuestro estudio (Tabla 5).

Tabla 5. Comparación de parámetros prequirúrgicos

\begin{tabular}{lrrrr} 
& \multicolumn{1}{c}{ Gea } & Leslee (USA) & \multicolumn{1}{c}{$\begin{array}{c}\text { Mordechai } \\
\text { Israel }\end{array}$} & $\begin{array}{c}\text { Khelifa } \\
\text { Francia }\end{array}$ \\
Edad & $42.7(25-56)$ & $46(37-54)$ & $41.3+/-11.5$ & $45.9+/-11.9$ \\
Peso (Kg) & $108.3(76-187)$ & 110 & & \\
IMC (Kg/m2) & $42.7(33.1-66.2)$ & $45.5(41.6-50.9)$ & $42.2+/-5.2$ & $43.7+/-6.9$ \\
IU (\%) & 73.1 & 49.3 & 37.7 & 50.9 \\
IUU (\%) & 32.7 & 33 & 7 & 36.8 \\
IUE (\%) & 55.1 & 42.2 & 59 & 39.7 \\
IUM (\%) & 12.2 & 24.8 & 34 & 23.5 \\
3 meses & $35.4+/-6.5$ & & & \\
6 meses & $31.9+/-5.8$ & 33 & & 30.2 \\
12 meses & $30.9+/-5.9$ & & & \\
IU (\%) & & & & \\
3 meses & 41.8 & 18.2 & & \\
6 meses & 17.9 & & & \\
12 meses & 13.4 & & & \\
\hline
\end{tabular}

En cuanto a la edad media de nuestra población fue de 42.7+/-7.9 años, menor a la reportada por Subak et al., ${ }^{(23)}$ de 46 años, pero similar a los 41.3 años reportados por Shimonov et al., ${ }^{(21)}$ el peso prequirúrgico promedio fue de 108.3 +/- $23.2 \mathrm{~kg}$ y el IMC de 42.7 +/- $8.3 \mathrm{~kg} / \mathrm{m} 2 \mathrm{me-}$ nor al de Said et al., ${ }^{(22)}$ de $43.7 \mathrm{~kg} / \mathrm{m} 2$ y Subak et al., ${ }^{(23)}$ de $45.5 \mathrm{~kg} / \mathrm{m} 2$ (Tabla 5).

Del total de nuestras pacientes ninguna contaba con cirugía pélvica previa para incontinencia.

El mayor tipo de incontinencia encontrado fue de esfuerzo con $55.1 \%$, parecido a lo repor- tado por Shimonov et al., ${ }^{(21)} 59 \%$, en segundo lugar la IUU en $32.7 \%$ y por último la IUM en $12.2 \%$. De hecho, Shimonov et al. ${ }^{(21)}$ encontró en segundo lugar IUM en $34 \%$ e IUU en último lugar (7\%); teniendo contraste con algunas otras series que reportaron en primer lugar la IUM en un $32 \%$, seguido de IUE en un $28 \%$ y por último IUU en $4 \% .{ }^{(21)}$ (Tabla 5).

El tipo de cirugía que más se realizó fue manga gástrica laparoscópica en 26 pacientes (53.1\%), seguida de bypass gástrico laparoscópico en 23 (46.9\%), y si bien a dos pacientes se les realizó bypass gástrico asistido por robot, 
dichas pacientes no presentaban Incontinencia Urinaria.

La mayor frecuencia de presentación fue de una vez por semana en 33 (67.3\%), la menor fue continua y varias veces al día en 2 (4.1\%), para cada una. La cantidad moderada fue menor en un $34.7 \%$ (17), seguido de poca en $65.3 \%$ (32. Ninguna paciente refirió perder mucha cantidad. La afección de la incontinencia en la calidad de vida solo se presentó en $30.6 \%$ (15), el resto de las pacientes no tenían repercusión al realizar sus actividades diarias ni de recreación.

La pérdida de peso promedio a los seis meses fue de $26.6+/-11.1 \mathrm{~kg}$, la recuperación del IMC fue de $10.7+/-4.4 \mathrm{~kg} / \mathrm{m} 2$ con una media de $31.9+/-5.8 \mathrm{~kg} / \mathrm{m} 2$, parecida a la reportada por Shimonov et al., ${ }^{(21)}$ de $33+/-4.7 \mathrm{~kg} / \mathrm{m} 2$.

A los tres meses de la cirugía disminuyó la prevalencia de IU a $41.8 \%$, persistiendo con mayor frecuencia la IUE $53.6 \%$, la frecuencia disminuyó al no presentar pérdidas continuas ni varias veces al día, persistiendo en primer lugar una vez a la semana en $46.4 \%$. La cantidad más encontrada siguió siendo poca en $78.6 \%$, hubo mejoría en la calidad de vida en 5 pacientes de las 15 afectadas al inicio del estudio.

A los 6 meses postquirúrgicos la prevalencia de la incontinencia fue de $17.9 \%$, se obtuvo una resolución completa de $75.5 \%(\mathrm{p}=0.02)$, mayor a $51.7 \%$ encontrada por Shimonov et al., (21) También hubo mejoría significativa en los tres tipos de incontinencia, frecuencia y calidad de vida con $\mathrm{p}=0.001, \mathrm{p}<0.001$ y $\mathrm{p}<0.001$ respectivamente, en comparación con lo estudiado por Shimonov et al., ${ }^{(21)}$ encontrando solo mejoría en la IU, los síntomas de llenado y la puntuación del cuestionario ICIQ para la calidad de vida $(\mathrm{p}<0.001)($ Tabla 5).
$\mathrm{Al}$ año de seguimiento la prevalencia de IU fue del $13.4 \%$, menor a la reportada por Subak et al., ${ }^{(23)}$ de $18.2 \%$, la menor frecuencia encontrada fue diariamente en $11.1 \%$, mayor que 8.4\% descrita por Subak et al., ${ }^{(23)}$ (Tabla 5).

\section{Conclusiones}

La obesidad es una enfermedad con alta repercusión en la Salud Pública, que ha estado en aumento en los últimos años. Es un factor de riesgo modificable para diferentes enfermedades crónico-degenerativas, principalmente cardiovasculares, así como para disfunción sexual, alteraciones en el piso pélvico e incontinencia urinaria de cualquier tipo (esfuerzo, urgencia y mixta), la cual aumenta los costos sanitarios.

Los resultados de este estudio permiten confirmar que la pérdida de peso después de la cirugía bariátrica conlleva a una resolución de la incontinencia urinaria en 81.6\%, 12 meses posteriores a la cirugía, y se presenta una mejoría estadísticamente significativa para los tres tipos de incontinencia (sobre todo para la IUU y la IUM), todo lo cual impacta favorablemente la calidad de vida en este tipo de pacientes. Por tanto se concluye que se debe considerar la reducción de peso como una terapia de primera línea para la Incontinencia Urinaria. Sin embargo, no encontramos ningún efecto significativo para la cantidad de orina pérdida en cada evento.

Aunque los objetivos principales de la cirugía bariátrica son disminuir las morbilidades cardiovasculares y la DM, la recuperación y/o mejoría de la incontinencia urinaria es un beneficio importante. Por tanto, debe evaluarse rutinariamente con cuestionarios validados, lo cual ofrece otra ventaja a largo plazo, pues 


\section{Análisis prospectivo de la obesidad y el efecto de la cirugía.... Calvo-Vázquez l., et al.}

ayuda a recuperar una mejor calidad de vida y motiva a las pacientes con obesidad e Incontinencia Urinaria a someterse a alguna cirugía de este tipo.

\section{Referencias}

1. Guh DP, Zhang W, Bansback N, Amarsi Z, Birmingham CL, Anis AH. The incidence of comorbidities related to obesity and overweight: a systematic review and meta-analysis. BMC Public Health. 2009;9:88. doi: https://doi. org/10.1186/1471-2458-9-88

2. Kelly T, Yang W, Chen C-S, Reynolds K, He J. Global burden of obesity in 2005 and projections to 2030. Int J Obes 2005. 2008;32(9):1431-7. doi: https://doi.org/10.1038/ijo.2008.102

3. Wang Y, Beydoun MA, Liang L, Caballero B, Kumanyika SK. Will all Americans become overweight or obese? estimating the progression and cost of the US obesity epidemic. Obes Silver Spring Md. 2008;16(10):2323-30. doi: https://doi.org/10.1038/oby.2008.351

4. Flegal KM, Carroll MD, Kit BK, Ogden CL. Prevalence of obesity and trends in the distribution of body mass index among US adults, 1999-2010. JAMA. 2012;307(5):491-7. doi: https://doi.org/10.1001/jama.2012.39

5. Mokdad AH, Ford ES, Bowman BA, Dietz WH, Vinicor F, Bales VS, et al. Prevalence of obesity, diabetes, and obesity-related health risk factors, 2001. JAMA. 2003;289(1):76-9.

6. Secretaría de Salud. Encuesta Nacional de Salud y Nutrición de Medio Camino 2016. 2016. [accessed 7 May 2019] Available from: http:// www.gob.mx/salud/documentos/encuestanacional-de-salud-y-nutricion-de-mediocamino-2016
7. World Health Organization. Obesity and overweight. World Health Organization. 2018. [accessed 24 Sep 2018] Available from: http:// www.who.int/news-room/fact-sheets/detail/ obesity-and-overweight

8. Waetjen LE, Liao S, Johnson WO, Sampselle CM, Sternfield B, Harlow SD, et al. Factors associated with prevalent and incident urinary incontinence in a cohort of midlife women: a longitudinal analysis of data: study of women's health across the nation. Am J Epidemiol. 2007 Feb 1;165(3):309-18. doi: https://doi. org/10.1093/aje/kwk018

9. Hunskaar S. A systematic review of overweight and obesity as risk factors and targets for clinical intervention for urinary incontinence in women. Neurourol Urodyn. 2008;27(8):74957. doi: https://doi.org/10.1002/nau.20635

10. Cundiff GW, Harris RL, Coates KW, Bump RC. Clinical predictors of urinary incontinence in women. Am J Obstet Gynecol. 1997;177(2):2626; discussion 266-267.

11. Withrow D, Alter DA. The economic burden of obesity worldwide: a systematic review of the direct costs of obesity. Obes Rev Off J Int Assoc Study Obes. 2011;12(2):131-41. doi: https:// doi.org/10.1111/j.1467-789X.2009.00712.x

12. Subak LL, Richter HE, Hunskaar S. Obesity and Urinary Incontinence: Epidemiology and Clinical Research Update. J Urol. 2009;182(6 Suppl):S2-7. [accessed 7 May 2019] Available from: https://www.ncbi.nlm.nih.gov/pmc/ articles/PMC2866035/

13. Pories WJ, Swanson MS, MacDonald KG, Long SB, Morris PG, Brown BM, et al. Who would have thought it? An operation proves to be the most effective therapy for adult-onset diabetes mellitus. Ann Surg. 1995;222(3):339-52. [accessed 7 May 2019] Available from: https://www.ncbi.nlm.nih. gov/pmc/articles/PMC1234815/ 
14. Hannestad YS, Rortveit G, Sandvik H, Hunskaar S, Norwegian EPINCONT study. Epidemiology of Incontinence in the County of Nord-Trøndelag. A community-based epidemiological survey of female urinary incontinence: the Norwegian EPINCONT study. Epidemiology of Incontinence in the County of Nord-Trøndelag. J Clin Epidemiol. 2000;53(11):1150-7.

15. Hunskaar S, Arnold EP, Burgio K, Diokno AC, Herzog AR, Mallett VT. Epidemiology and natural history of urinary incontinence. Int Urogynecol $\mathrm{J}$ Pelvic Floor Dysfunct. 2000;11(5):301-19.

16. Martínez-Espinoza CJ, Flores-Carreras Ó, García de Alba-García JE, VelázquezCastellanos PI, González-Ruiz MI, MárquezAllegre R. Prevalencia de incontinencia urinaria $\mathrm{y}$ anal en mujeres de la zona metropolitana de Guadalajara. Ginecol Obstet México. 2006;74(06):300-5. [accessed 7 May 2019] Available from: https://www.medigraphic.com/ cgi-bin/new/resumen.cgi?IDARTICULO=14478

17. Haylen BT, de Ridder D, Freeman RM, Swift SE, Berghmans B, Lee J, et al. An International Urogynecological Association (IUGA)/ International Continence Society (ICS) joint report on the terminology for female pelvic floor dysfunction. Int Urogynecology J. 2010;21(1):5-26. doi: https://doi.org/10.1007/ s00192-009-0976-9

18. Kammerer-Doak D, Rizk DEE, Sorinola O, Agur W, Ismail S, Bazi T. Mixed urinary incontinence: international urogynecological association research and development committee opinion. Int Urogynecology J. 2014;25(10):1303-12. doi: https://doi.org/10.1007/s00192-014-2485-8
19. Esin S, Salman MC, Ozyuncu O, Durukan T. Surgical outcome of transobturator tape procedure in obese and non-obese women. J Obstet Gynaecol J Inst Obstet Gynaecol. 2011;31(7):645-9. doi: https://doi.org/10.310 9/01443615.2011.597461

20. Yonguc T, Aydogdu O, Bozkurt IH, Degirmenci T, Gunlusoy B, Sen V, et al. Do severe obese patients with stress urinary incontinence benefit from transobturator tape procedure? 3-year surgical outcome. Can Urol Assoc J. 2015;9(7-8):E546-50. [accessed 7 May 2019] Available from: https://www.ncbi.nlm.nih.gov/ pmc/articles/PMC4641875/

21. Shimonov M, Groutz A, Schachter P, Gordon D. Is bariatric surgery the answer to urinary incontinence in obese women? Neurourol Urodyn. 2017;36(1):184-7. doi: https://doi. org/10.1002/nau.22909

22. Said KA, Leroux Y, Menahem B, Doerfler A, Alves A, Tillou X. Effect of bariatric surgery on urinary and fecal incontinence: prospective analysis with 1-year follow-up. Surg Obes Relat Dis. 2017;13(2):305-12. [accessed 7 May 2019] Available from: https://www.soard.org/article/ S1550-7289(16)30191-5/abstract

23. Subak LL, King WC, Belle SH, Chen J-Y, Courcoulas AP,EbelFE, etal.Urinary Incontinence Before and After Bariatric Surgery. JAMA Intern Med. 2015;175(8):1378-87. doi: https://doi. org/10.1001/jamainternmed.2015.2609

24. Sugerman H, Windsor A, Bessos M, Kellum J, Reines H, DeMaria E. Effects of surgically induced weight loss on urinary bladder pressure, sagittal abdominal diameter and obesity comorbidity. Int J Obes Relat Metab Disord J Int Assoc Study Obes. 1998;22(3):230-5. 


\section{Análisis prospectivo de la obesidad y el efecto de la cirugía.... Calvo-Vázquez l., et al.}

25. Cummings JM, Rodning CB. Urinary stress incontinence among obese women: review of pathophysiology therapy. Int Urogynecol J Pelvic Floor Dysfunct. 2000;11(1):41-4.

26. Subak LL, Whitcomb E, Shen H, Saxton J, Vittinghoff E, Brown JS. Weight loss: a novel and effective treatment for urinary incontinence. J
Urol. 2005;174(1):190-5. doi: https://dx.doi. org/10.1097\%2F01.ju.0000162056.30326.83

27. Osborn DJ, Strain M, Gomelsky A, Rothschild J, Dmochowski R. Obesity and female stress urinary incontinence. Urology. 2013;82(4):759-63. doi: https://doi.org/10.1016/j.urology.2013.06.020 